\title{
Synthesis and bioassay of $\beta-(1,4)-D$-mannans as potential agents against Alzheimer's disease
}

\author{
Ru-wei JIANG, Xiao-guang DU, Xuan ZHANG, Xin WANG, Ding-yu HU, Tao MENG, Yue-lei CHEN, Mei-yu GENG* , Jing-kang \\ SHEN*
}

State Key Laboratory of Drug Research, Shanghai Institute of Materia Medica, Shanghai 201203, China

\begin{abstract}
Aim: Oligomannurarate 971 derived from a marine plant has shown neuroprotective effects. In this study we synthesized a series of truncated derivatives of the oligosaccharide, and investigated the effect of these derivatives against $A \beta$ peptide toxicity in vitro. Methods: The sulfoxide method was applied to synthesize the derivatives. SH-SY5Y human neuroblastoma cells were treated with $A \beta_{1-40}(2 \mu \mathrm{mol} / \mathrm{L})$, and the cell viability was detected using a CCK8 assay.

Results: A series of $\beta-(1,4)-D$-mannosyl oligosaccharide, ranging from the disaccharide to the hexasaccharide, were synthesized. Addition of $10 \mu \mathrm{mol} / \mathrm{L} \beta$-(1,4)-D-mannobiose $6, \beta-(1,4)$-D-mannotriose 9 or $\beta$-(1,4)-D-mannotetraose 12 in SH-SY5Y cells significantly attenuated AB1-40-induced toxicity. The efficacies were similar to those caused by $10 \mu \mathrm{mol} / \mathrm{L}$ oligomannurarate 971 or alzhemed. Other oligosaccharides including oligomaltoses and oligocelluloses were less active.

Conclusion: Synthetic homogeneous short chain $\beta-(1,4)-D$-mannans shows neuroprotective effect against $A \beta$ peptide toxicity similar to that of heterogeneous oligomannurarate 971 and alzhemed.
\end{abstract}

Keywords: Alzheimer's disease; $A \beta$ peptide; oligosaccharide; $\beta-(1,4)-D-m a n n a n ;$ oligomannurarate 971; alzhemed; neuroprotection; medicinal chemistry

Acta Pharmacologica Sinica (2013) 34: 1585-1591; doi: 10.1038/aps.2013.104; published online 18 Nov 2013

\section{Introduction}

Alzheimer's disease (AD) is a neurodegenerative disease with multiple etiologies. As no effective treatments have yet been developed, AD remains pandemic in the 21st century and imposes enormous social and economic burdens on patients and their families ${ }^{[1]}$. It is estimated that 5.4 million individuals in the United States suffer from the disease, with AD patients numbering as many as 30 million globally, and these numbers continue to increase $\mathrm{e}^{[2]}$.

$\mathrm{AD}$ is characterized histopathologically by senile plaques, neurofibrillary tangles, reactive astrocytosis and neuronal cell loss. The $A \beta$ peptide, the major component of senile plaques, has been identified in numerous cases as a major causative factor in $\mathrm{AD}$ pathophysiology ${ }^{[3,4]}$. The $\mathrm{A} \beta$ peptide has, consequently, become one of the most important targets for $\mathrm{AD}$ therapy.

Carbohydrate drugs are widely employed in the treatment of a number of major diseases owing to their varied bioactivi-

\footnotetext{
* To whom correspondence should be addressed.

E-mail mygeng@simm.ac.cn (Mei-yu GENG); jkshen@mail.shcnc.ac.cn (Jing-kang SHEN)

Received 2013-04-14 Accepted 2013-07-17
}

ties and low toxicity ${ }^{[5-10]}$. Certain types of saccharides have also been shown to demonstrate neuroprotective effects ${ }^{[9-10]}$, but the limited availability of pure saccharide compounds restricts further research on their activity. However, oligomannurarate 971 is a readily available, inexpensive and potent neuroprotective drug. Derived from a marine plant, the acidic heterogeneous $\beta-(1,4)$-D-oligomannurarate 971 (Figure 1) is a potent inhibitor of neurotoxicity induced by the $A \beta$ peptide. It is believed that 971 penetrates the blood brain barrier with the aid of the transporter GLUT1, and it exhibits better efficacy in animal models of dementia than does alzhemed (also known as $\mathrm{AZ}$, an anti-AD drug targeting the HHQK subregion at the $\mathrm{N}$-terminus of $A \beta_{1-40}$, which failed in a late stage Phase III clinical trial $)^{[11,12]}$. Mannurarate 971 is currently undergoing Phase II clinical studies ${ }^{[12]}$. As synthetic medicinal chemists, we wondered if truncated derivatives of 971 would exhibit similar

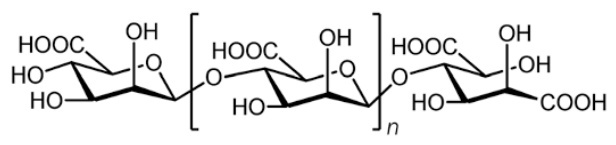

Figure 1. The structure of oligomannurarate $971 . n=2-8$. 
activity. We focused specifically on $\beta-(1,4)-D$-mannans as simplified 971 analogs. To the best of our knowledge, the neuroprotective effects of $\beta-(1,4)-D$-mannans have not been reported in the literature. This may be due to the limited availability of these compounds. While it can be very difficult to synthesize pure $\beta$-(1,4)-D-mannans, an effective solution was reported recently by Crich et al ${ }^{[13-17]}$. We followed the reported method and prepared a series of $\beta-(1,4)-D$-mannans for biological studies.

\section{Materials and methods Chemistry}

The sulfoxide method ${ }^{[13-17]}$ is one of the few approaches that can be used reliably to overcome the difficulties in controlling anomeric stereochemistry and yield the desired $\beta-(1,4)-D$ mannosyl oligosaccharide series. We have used this method to prepare the target $\beta-(1,4)-D$-mannosyl oligosaccharide series, ranging from the disaccharide to the hexasaccharide, via multistep sequences. The linear syntheses of $\beta$-mannans commenced from the sulfoxide glycoside donor $\mathbf{1}^{[18]}$, which was prepared in 6 steps from $D$-mannose ( $30 \%$ yield overall). Activation of mannose donor 1 at $-78^{\circ} \mathrm{C}$ with triflic anhydride $\left(\mathrm{Tf}_{2} \mathrm{O}\right)$ in the presence of the hindered base 2,4,6-tri-tert-butylpyrimidine (TTBP) $)^{[17]}$, followed by addition of benzyl alcohol, provided benzyl $\beta$-mannoside $2^{[19]}$. Removal of the benzylidene acetal of $\mathbf{2}$ under acidic conditions yielded the intermediate diol (structure not shown), which was acetylated regioselectively at the 6-OH to give mannosyl acceptor 3. Coupling of acceptor $\mathbf{3}$ with mannosyl donor $\mathbf{1}$ under Crich's standard conditions afforded the fully protected $\beta$-(1,4)-linked mannosyl disaccharide 4 in $70 \%$ yield (Scheme 1). The $\beta$-configuration of 4 was confirmed from the assignment of the H-5 chemical shift at $\delta 3.09^{[18]}$ in the ${ }^{1} \mathrm{H}$ NMR spectrum. This peak appeared as a doublet of triples, which is typical and diagnostic for the $\beta$-configuration in 4,6-O-benzylidene-protected mannosides. Glycoside 4 also displayed two anomeric carbon signals at $\delta$ 101.4 and $\delta 99.8$ with ${ }^{1} \mathrm{~J}_{\mathrm{CH}}$ coupling constants of 162.5 and 155 $\mathrm{Hz}$, respectively, a pattern that is consistent with the presence of $\beta$-O-glycosides ${ }^{[20]}$. The anomeric stereochemistry in all subsequent coupling products was assigned by similar comparison of the $\mathrm{H}-5$ chemical shift in the ${ }^{1} \mathrm{H}$ NMR spectrum.

The preparation of $\beta$-(1,4)-linked mannosyl disaccharide 4 from $\beta$-mannoside 2 represented the first glycosylation cycle of a two-stage iterative sequence ${ }^{[17]}$. This protocol was repeated to provide the fully protected $\beta$-(1,4)-mannans $7, \mathbf{1 0}$, 13, and 16. Deacetylation of the corresponding acceptor followed by global debenzylation under catalytic hydrogenation conditions furnished the corresponding $\beta-(1,4)$-D-mannans 6 ,
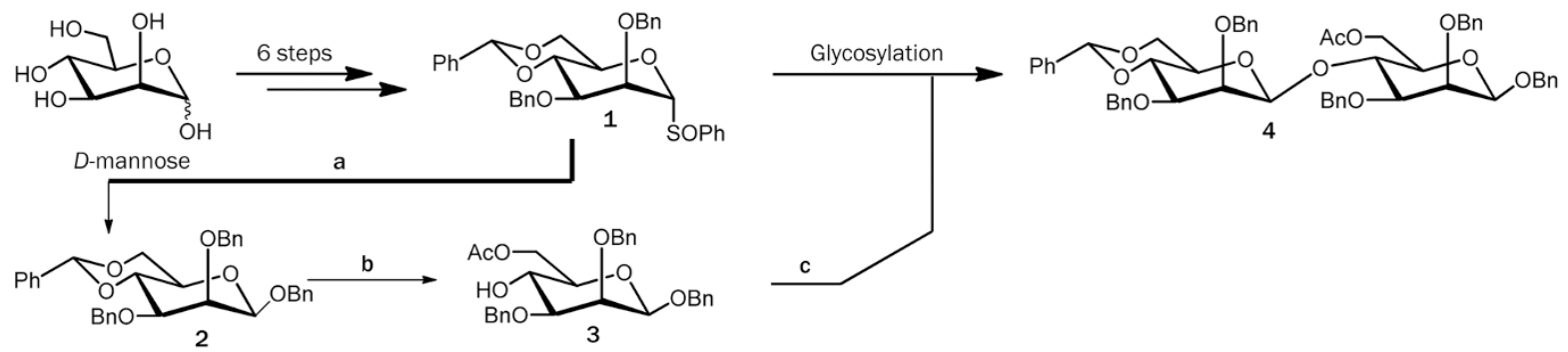

Scheme 1. Synthesis of compound 4. Reagents and conditions: (a) $\operatorname{Tf}_{2} \mathrm{O}, \mathrm{TTBP}, \mathrm{BnOH}$; (b) TFA then $\mathrm{Ac}_{2} \mathrm{O}, \mathrm{Et}_{3} \mathrm{~N}$; (c) $\mathrm{Tf}_{2} \mathrm{O}, \mathrm{TTBP}_{\text {. }}$

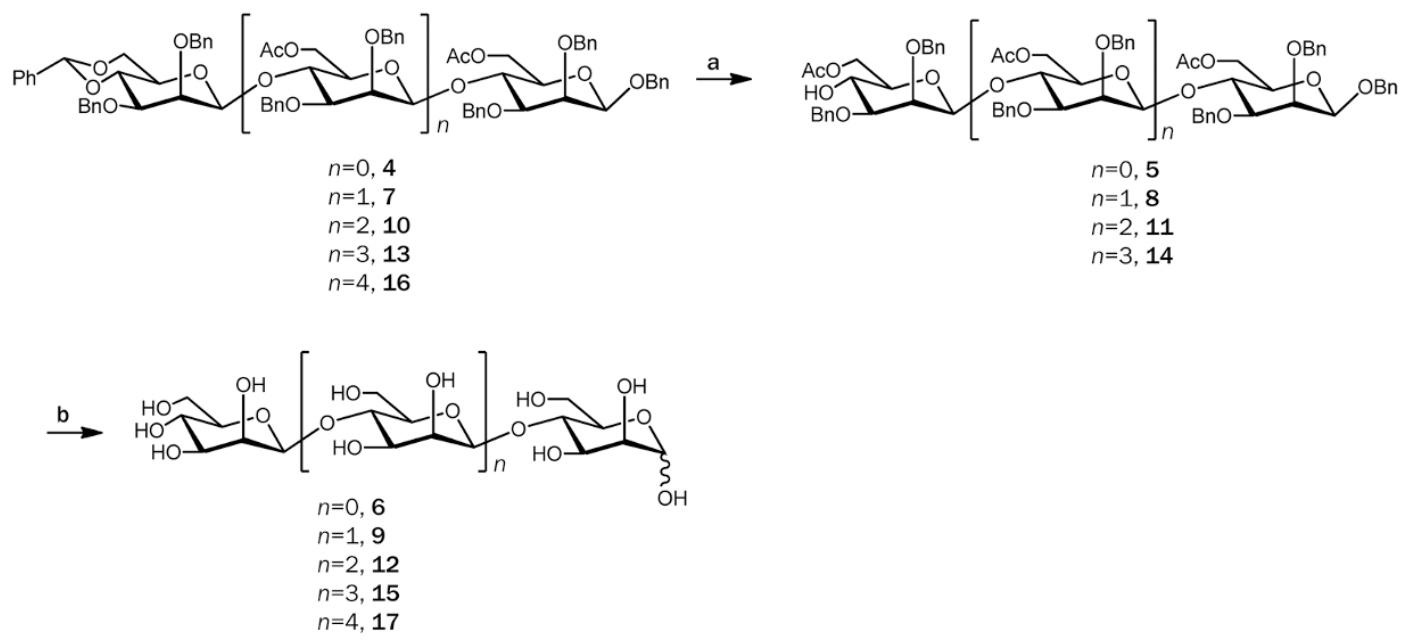

Scheme 2. Synthesis of compounds 6, 9, 12, 15, and 17. Reagents and conditions: (a) TFA, then $\mathrm{Ac}_{2} \mathrm{O}, \mathrm{Et}_{3} \mathrm{~N}$; $(\mathrm{b}) \mathrm{K}_{2} \mathrm{CO}_{3}, \mathrm{MeOH}$ then $20 \% \mathrm{Pd}(\mathrm{OH})_{2} / \mathrm{C}$, $\mathrm{H}_{2}$. Compound 17 was obtained directly from 16 using conditions (b). 
9, 12, 15, and 17 (Scheme 2).

\section{Drugs and reagents}

The $\beta$ - $(1,4)-D$-mannosyl oligosaccharide series was synthesized in our laboratory. Oligomannurarate 971 was obtained from Mei-yu GENG's lab at the Shanghai Institute of Materia Medica. Alzhemed (AZ) was purchased from Sigma-Aldrich China and the maltose and cellulose series were purchased from J\&K Chemical Ltd (Shanghai, China).

\section{Neuroprotective effect assay against $A \beta$ peptide toxicity}

SH-SY5Y human neuroblastoma cells were purchased from the American Type Culture Collection (ATCC, Manassas, VA, USA). These are stable cells that typically will not be differentiated by exposure to small carbohydrates. Cells were plated at 4000 cells per well in 96-well plates and cultured in Dulbecco's modified Eagle's medium at $37^{\circ} \mathrm{C}$ in $5 \% \mathrm{CO}_{2}(v / v)$ in a humidified incubator. After $24 \mathrm{~h}$, aged $\mathrm{A} \beta_{1-40}$ (incubated for 96 h) was added to cells in combination with various compounds at different concentrations and the cells were incubated for 48 h. The final concentration of $\mathrm{A} \beta$ was $2 \mu \mathrm{mol} / \mathrm{L}$. Cell viability was measured in a CCK8 assay.

\section{Statistical analysis}

The data were expressed as mean \pm SD. The Student's $t$-test was used for statistical analysis.

\section{Results and discussion}

Neuroprotective effects of oligosaccharides against $A \beta$ peptide toxicity

The synthetic homogeneous $\beta-(1,4)-D$-mannosyl oligosaccharides were assessed for activity against $A \beta$ peptide neurotoxicity. Control compounds included $D$-mannose, mannurarate 971 and AZ. Commercially available oligomaltoses, including maltobiose (M2), maltotriose (M3), maltotetraose (M4), maltopentaose (M5), maltohexaose (M6), and maltoheptaose (M7) were assessed for comparison with the synthetic compounds. Oligocelluloses, including cellobiose (C2), cellotriose (C3), cellotetraose (C4), cellopentaose (C5) and cellohexose (C6) were also assessed.

Mannurarate $971(O D=1.31)$ and $A Z(O D=1.37)$ were found to exhibit potent neuroprotective effects ( $\mathrm{A} \beta$ model: $O D=1.12$, control: $O D=1.42 \pm 0.02)$ as expected. $\mathrm{AZ}$ was the most potent of the compounds tested. The synthetic homogeneous $\beta-(1,4)$ $D$-mannosyl oligosaccharides $6(O D=1.32), 9(O D=1.35), 12$ $(O D=1.27), 15(O D=1.22)$, and $17(O D=1.24)$ also showed neuroprotective potency. Compounds 6 and 9 were slightly more active than 971 and were nearly as potent as AZ. This is a significant result in light of the structural simplicity of these compounds. D-mannose did not show any neuroprotective activity. Oligocelluloses and oligomaltoses were also examined to shed further light on the neuroprotective activity of oligosaccharides. Only maltoheptaose (M7) showed significant activity $(O D=1.27)$, while the others were largely inactive (M2: $O D=1.23$; M3: $O D=1.17$; $M 4: O D=1.17$; $\mathrm{M} 5: O D=1.19$; M6: $O D=1.22 ; C 2: O D=1.17 ; C 3: O D=1.11 ; C 4: O D=1.15 ; C 5$ :

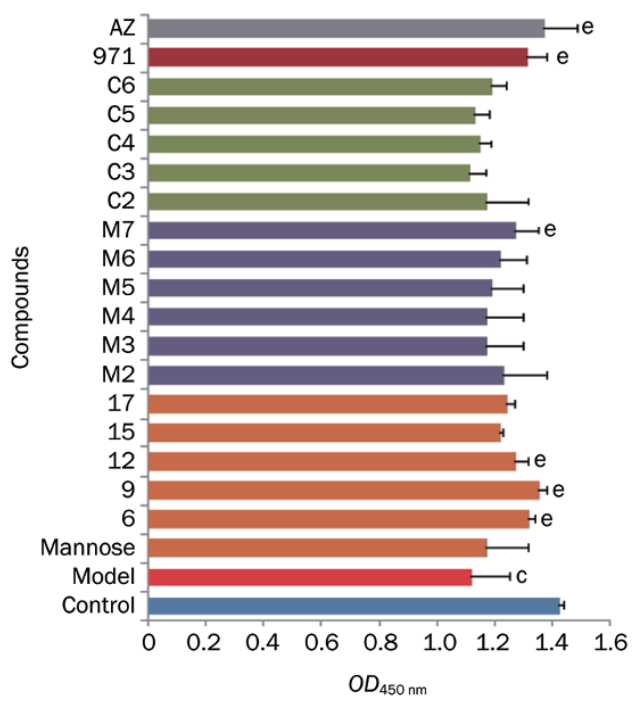

Figure 2. Neuroprotective effects of oligosaccharides against $A \beta$ peptide toxicity on SH-SY5Y cells (mean \pm SD, $n=3$ ). ${ }^{\mathrm{c}} P<0.01$ vs control. ${ }^{\mathrm{e}} P<0.05$ vs model. M2-M7 represent maltobiose-maltoheptaose, respectively. C2-C6 represent cellobiose-cellohexose, respectively. The concentrations of 6-17, M2-M7, and C2-C6 were $10 \mu \mathrm{mol} / \mathrm{L}$; the concentration of 971 was $50 \mu \mathrm{g} / \mathrm{mL}$ (approximately $50 \mu \mathrm{mol} / \mathrm{L}$ ); the concentration of $\mathrm{AZ}$ was 50 $\mu \mathrm{mol} / \mathrm{L}$.

$O D=1.13$; $C 6: O D=1.19$ ) (Figure 2). The differences in activity between oligomannoses, oligomaltoses and oligocellulose indicate that the nature of the monosaccharide unit and the configuration of the anomeric center exert significant influence over the neuroprotective potency of oligosaccharides. We believe that this preliminary finding reveals important information on the structural characteristics of oligosaccharides that exhibit neuroprotective effects. More data are required to enable a detailed analysis of structure activity relationships.

\section{Conclusion}

In this study, we applied the sulfoxide method to the preparation of a series of $\beta$ - $(1,4)-D$-mannosyl oligosaccharides. Oligosaccharides ranging from disaccharide to hexasaccharide were synthesized in multistep sequences. The neuroprotective activity of synthetic mannans was assessed and compared with that of mannose, 971, AZ and a series of commercially available oligomaltoses and oligocelluloses. Synthetic compounds $\beta$-(1,4)-D-mannobiose 6, $\beta$-(1,4)-D-mannotriose 9 and $\beta$-(1,4)-D-mannotetraose 12 showed potency similar to that of 971 (and were slightly less potent than AZ) as inhibitors of toxicity induced by the $A \beta$ peptide. Other oligosaccharides failed to show significant neuroprotective activity. Taken together, these results demonstrate that the structure of 971 can be modified without a loss of activity. We have disclosed a new class of neuroprotective agents with potency against $A \beta$ toxicity and gained insight that will enable development of potent agents for the treatment of Alzheimer's disease. Further research is ongoing in our group. 


\section{Experiment}

Reagents (chemicals) were purchased from Acros (Geel, Belgium) and the Shanghai Chemical Reagent Company (Shanghai, China) and were used without purification. Analytical thin-layer chromatography was performed on HSGF 254 plates (150-200 $\mu \mathrm{m}$ thickness; Yantai Huiyou Company, Yantai, Shandong, China). ${ }^{1} \mathrm{H}$ NMR (300 MHz or $\left.400 \mathrm{MHz}\right)$ spectra were recorded on Varian Mercury-300 or 400 High Performance Digital FT-NMR(Varian, Fort Collins, CO, USA) instruments using TMS as the internal standard. ${ }^{13} \mathrm{C}$ NMR $(100 \mathrm{MHz})$ spectra were determined using a Varian Mercury-400 High Performance Digital FT-NMR. Chemical shifts are reported in parts per million (ppm, $\delta$ ) downfield from tetramethylsilane. Proton coupling patterns are described as singlet $(\mathrm{s})$, doublet $(\mathrm{d})$, triplet $(\mathrm{t})$, quartet $(\mathrm{q})$, multiplet $(\mathrm{m})$, and broad (br). LC-MS analysis was carried out on a Thermo Finnigan LCQ Deca XP (Thermo Electron Corporation, San Jose, CA, USA) and HRMS was performed on a Finnigan MAT 95 mass spectrometer (Thermo Fisher Scientific, Waltham, Massachusetts, USA). Optical rotation values were determined using a PerkinElmer-341 (589 nm) polarimeter (PerkinElmer, Waltham, Massachusetts, USA).

\section{General procedure for preparation of $\beta$-mannosides by the sulfoxide method}

A dispersion of sulfoxide, TTBP (2.5 equiv.) and powdered molecular sieves in $\mathrm{CH}_{2} \mathrm{Cl}_{2}(0.1 \mathrm{~mol} / \mathrm{L})$ was cooled to $-78^{\circ} \mathrm{C}$ for $30 \mathrm{~min}$. To this mixture was added a solution of $\mathrm{Tf}_{2} \mathrm{O}(1$ $\mathrm{mol} / \mathrm{L}$ in $\mathrm{CH}_{2} \mathrm{Cl}_{2}, 1.1$ equiv.) dropwise at $-78^{\circ} \mathrm{C}$. The acceptor (1.2 equiv.) was added slowly as a solution in $\mathrm{CH}_{2} \mathrm{Cl}_{2}(1.0$ $\mathrm{mol} / \mathrm{L}$ ). The reaction mixture was stirred at $-78^{\circ} \mathrm{C}$ for $1 \mathrm{~h}$, then gradually warmed to $-20^{\circ} \mathrm{C}$ while the stirring was continued. When TLC indicated complete consumption of the sulfoxide, the reaction was quenched with saturated aq. $\mathrm{NaHCO}_{3}$. The aqueous phase was extracted thrice with EtOAc. The combined organic phases were washed with water and brine, dried over anhydrous $\mathrm{Na}_{2} \mathrm{SO}_{4}$, filtered and concentrated. The residue was purified by silica gel chromatography.

\section{Benzyl 2,3-di-0-benzyl-6-0-acetyl- $\beta$-D-mannopyranoside (3)}

To a solution of compound $2(14.86 \mathrm{~g}$, $27.62 \mathrm{mmol})$ in $\mathrm{CH}_{2} \mathrm{Cl}_{2}$ $(200 \mathrm{~mL})$ was added TFA $/ \mathrm{H}_{2} \mathrm{O}(85.2 \mathrm{~mL}, 1: 1 \mathrm{v} / \mathrm{v})$ slowly at $0^{\circ} \mathrm{C}$. The mixture was stirred at room temperature for $1 \mathrm{~h}$ until TLC showed complete consumption of 2 . The mixture was diluted with $\mathrm{CH}_{2} \mathrm{Cl}_{2}$, the reaction quenched with cold saturated $a q . \mathrm{NaHCO}_{3}$ and the organic layer was separated. The aqueous phase was extracted thrice with $\mathrm{CH}_{2} \mathrm{Cl}_{2}$. The combined organic phases were washed with saturated aq. $\mathrm{NaHCO}_{3}$ and brine, dried over anhydrous $\mathrm{Na}_{2} \mathrm{SO}_{4}$ and concentrated. The residue was taken up in a mixture of $\mathrm{CH}_{2} \mathrm{Cl}_{2}(200$ $\mathrm{mL}$ ) and TEA $(9.6 \mathrm{~mL}, 69.05 \mathrm{mmol})$, and to this solution was added $\mathrm{Ac}_{2} \mathrm{O}(6.5 \mathrm{~mL}, 552.42 \mathrm{mmol})$. The mixture was stirred at room temperature for $4 \mathrm{~h}$. When TLC showed complete consumption of the starting material, the reaction was quenched with saturated $a q . \mathrm{NaHCO}_{3}$ and diluted with $\mathrm{CH}_{2} \mathrm{Cl}_{2}$. The phases were separated and the aqueous phase was extracted twice with $\mathrm{CH}_{2} \mathrm{Cl}_{2}$. The combined organic phases were dried over anhydrous $\mathrm{Na}_{2} \mathrm{SO}_{4}$, filtered and concentrated in vacuo. The residue was purified by silica gel column chromatography to give the title compound $(6.3 \mathrm{~g}, 46 \%)$ as a colorless oil. ${ }^{1} \mathrm{H}$ NMR (300 MHz, $\left.\mathrm{CDCl}_{3}\right) \delta$ 7.44-7.21 (m, 15H, ArH), 4.99 (d, $\left.\mathrm{J}=11.9 \mathrm{~Hz}, 2 \mathrm{H}, \mathrm{PhCH}_{2}\right), 4.80\left(\mathrm{~d}, \mathrm{~J}=12.4 \mathrm{~Hz}, 1 \mathrm{H}, \mathrm{PhCH}_{2}\right), 4.61$ (d, J=12.0 Hz, 1H, $\left.\mathrm{PhCH}_{2}\right), 4.51-4.39$ (m, 4H, $\mathrm{PhCH}_{2}, \mathrm{H} 1, \mathrm{H} 6 \mathrm{a}$, H6b), 4.29 (d, J=11.8 Hz, 1H, $\left.\mathrm{PhCH}_{2}\right), 3.99-3.87$ (m, 2H, H4, H2), 3.40 (ddd, J=9.6, 4.9, $3.1 \mathrm{~Hz}, 1 \mathrm{H}, \mathrm{H} 5), 3.27$ (dd, J=9.4, 3.0 $\mathrm{Hz}, 1 \mathrm{H}, \mathrm{H} 3), 2.55$ (s, 1H, OH), 2.12 (s, 3H, OAc). ${ }^{13} \mathrm{C} \mathrm{NMR} \mathrm{(100}$ $\left.\mathrm{MHz}, \mathrm{CDCl}_{3}\right): \delta=171.3,138.4,137.5,137.1,128.4-127.4,100.2$, 81.2, 74.2, 74.0, 73.1, 71.1, 70.7, 66.6, 63.8, 20.8. ESI-MS: $\mathrm{m} / \mathrm{z}$ $515.3[\mathrm{M}+\mathrm{Na}]^{+}$. HRMS: calcd for $\mathrm{C}_{29} \mathrm{H}_{32} \mathrm{O}_{7} \mathrm{Na} 515.2046$, found: 515.2040. $[\mathrm{\alpha}]_{\mathrm{D}}{ }^{21}=-110,\left(\mathrm{c} 0.5, \mathrm{CHCl}_{3}\right)$.

Benzyl 2,3-di-O-benzyl-6-acetyl-4-0-(2,3-di-0-benzyl-4,6-0-benzylidene- $\beta$-D-mannopyranosyl)- $\beta$ - $D$-mannopyranoside (4)

Coupling sulfoxide $\mathbf{1}$ with $\mathbf{3}$ under the standard sulfoxide $\beta$-mannosylation conditions afforded the title compound in $65 \%$ yield as a colorless oil. ${ }^{1} \mathrm{H}$ NMR $\left(400 \mathrm{MHz}, \mathrm{CDCl}_{3}\right) \delta$ 7.49-7.20 (m, 30H, ArH), 5.52 (s, 1H, PhCH), 5.01-4.86 (m, 3H, $\left.\mathrm{PhCH}_{2}\right), 4.84-4.72\left(\mathrm{~m}, 3 \mathrm{H}, \mathrm{PhCH}_{2}\right), 4.68-4.55\left(\mathrm{~m}, 4 \mathrm{H}, \mathrm{PhCH}_{2}\right.$, $\mathrm{H} 1), 4.50\left(\mathrm{~d}, \mathrm{~J}=11.9 \mathrm{~Hz}, 1 \mathrm{H}, \mathrm{PhCH}_{2}\right), 4.46\left(\mathrm{~s}, 1 \mathrm{H}, \mathrm{H1} 1^{\prime}\right), 4.31$ (m, 2H, H6a, H6b), 4.14-4.03 (m, 2H, H4, H6a'), 3.99 (dd, J=10.4, $\left.4.8 \mathrm{~Hz}, 1 \mathrm{H}, \mathrm{H}_{6} \mathrm{~b}^{\prime}\right), 3.90\left(\mathrm{~m}, 2 \mathrm{H}, \mathrm{H} 2, \mathrm{~Hz}{ }^{\prime}\right), 3.66(\mathrm{t}, \mathrm{J}=10.2 \mathrm{~Hz}, 1 \mathrm{H}$, $\left.\mathrm{H} 4^{\prime}\right)$, 3.58-3.49 (m, 2H, H3, H3'), 3.49-3.43 (m, 1H, H5), 3.09 $\left(\mathrm{td}, \mathrm{J}=9.9,4.9 \mathrm{~Hz}, 1 \mathrm{H}, \mathrm{H5}{ }^{\prime}\right), 2.08$ (s, 3H, OAc). ${ }^{13} \mathrm{C}$ NMR $(100$ $\left.\mathrm{MHz}, \mathrm{CDCl}_{3}\right): \delta=170.8,138.6,138.5,138.4,137.6,137.1,128.8$, 128.4-127.1, 126.1, 101.7, 101.4, 99.8, 79.6, 78.6, 78.3, 75.7, 75.2, 74.1, 74.0, 73.4, 72.6, 71.7, 70.8, 68.5, 67.4, 63.5, 21.0. ESI-MS: $\mathrm{m} / \mathrm{z} 945.6[\mathrm{M}+\mathrm{Na}]^{+}$. HRMS: calcd for $\mathrm{C}_{56} \mathrm{H}_{58} \mathrm{O}_{12} \mathrm{Na}$ 945.3826, found: 945.3823 . $[\mathrm{\alpha}]_{\mathrm{D}}{ }^{21}=-58.6,\left(\mathrm{c} 1, \mathrm{CHCl}_{3}\right)$.

Benzyl 2,3-di-0-benzyl-6-acetyl-4-0-(2,3-di-0-benzyl-6-0-acetyl- $\beta$ $D$-mannopyranosyl)-B-D-mannopyranoside (5)

The method used to prepare 3 was employed for the synthesis of the title compound (colorless oil, 70\% yield) using compound 4 as the starting material. ${ }^{1} \mathrm{H}$ NMR $\left(400 \mathrm{MHz}, \mathrm{CDCl}_{3}\right)$ $\delta 7.30$ (ddd, J=25.2, 15.9, 7.7 Hz, 25H, ArH), 5.00-4.89 (m, 2H, $\left.\mathrm{PhCH}_{2}\right), 4.86-4.71\left(\mathrm{~m}, 3 \mathrm{H}, \mathrm{PhCH}_{2}\right), 4.56\left(\mathrm{~m}, 5 \mathrm{H}, \mathrm{PhCH}_{2}\right), 4.44$ $\left(\mathrm{m}, 4 \mathrm{H}, \mathrm{H1}, \mathrm{H1} 1^{\prime}, \mathrm{H6a}, \mathrm{H} 6 \mathrm{~b}\right), 4.17$ (m, 2H, H6a', H6b'), 4.10 (t, $\mathrm{J}=8.9 \mathrm{~Hz}, 1 \mathrm{H}, \mathrm{H} 4), 3.90\left(\mathrm{~m}, 2 \mathrm{H}, \mathrm{H} 2, \mathrm{H} 2^{\prime}\right), 3.83$ (t, J=9.4 Hz, 1H, $\left.\mathrm{H} 4^{\prime}\right), 3.62-3.53(\mathrm{~m}, 2 \mathrm{H}, \mathrm{H} 3, \mathrm{H} 5), 3.20\left(\mathrm{~m}, 1 \mathrm{H}, \mathrm{H} 3^{\prime}\right), 3.10(\mathrm{~m}, 1 \mathrm{H}$, $\left.\mathrm{H}^{\prime}\right), 2.49$ (s, 1H, OH), 2.09 (s, 3H, OAc), 1.91 (s, 3H, OAc). ${ }^{13} \mathrm{C}$ NMR $\left(100 \mathrm{MHz}, \mathrm{CDCl}_{3}\right): \delta=171.1,170.8,138.6,138.4,138.2$, 137.6, 137.1, 128.5-127.2, 100.9, 99.8, 81.3, 78.9, 75.0, 74.4, 74.3, 74.1, 73.9, 73.8, 73.3, 71.3, 71.2, 70.7, 66.3, 63.6, 63.4, 20.9, 20.7. ESI-MS: $m / z 899.5[\mathrm{M}+\mathrm{Na}]^{+}$. HRMS: calcd for $\mathrm{C}_{51} \mathrm{H}_{56} \mathrm{O}_{13} \mathrm{Na}$ 899.3619, found: 899.3646. [a $]_{\mathrm{D}}{ }^{21}=-87.3,\left(\mathrm{c} 1, \mathrm{CHCl}_{3}\right)$.

\section{$\beta-(1,4)-D$-mannobiose $(6)^{[21]}$}

To a solution of compound 5 (79 $\mathrm{mg}, 0.1 \mathrm{mmol})$ in anhydrous $\mathrm{MeOH}(2.5 \mathrm{~mL})$ was added powdered $\mathrm{K}_{2} \mathrm{CO}_{3}(13.8 \mathrm{mg}, 0.1$ $\mathrm{mmol}$ ), and the mixture was stirred at room temperature for $1 \mathrm{~h}$. When TLC showed complete consumption of 5 , the mixture was filtered to remove solids and concentrated. The 
residue was taken up in $\mathrm{MeOH}(5 \mathrm{~mL})$, and to this solution was added $\mathrm{Pd}(\mathrm{OH})_{2} / \mathrm{C}(16 \mathrm{mg}, 20 \% \mathrm{Pd} w / w)$. The mixture was stirred under an atmosphere of $\mathrm{H}_{2}$ for $24 \mathrm{~h}$. The mixture was filtered through a Celite pad and concentrated. The residue was dissolved in water and purified by Sephadex LH20 gel chromatography eluted with water. The collected fractions were lyophilized to give the title compound (18 $\mathrm{mg}, 58 \%)$ as a white amorphous solid. ${ }^{1} \mathrm{H}$ NMR $\left(400 \mathrm{MHz}, \mathrm{D}_{2} \mathrm{O}\right) \delta 5.23(\mathrm{~s}$, $0.68 \mathrm{H}), 4.96(\mathrm{~s}, 0.32 \mathrm{H}), 4.79(\mathrm{~s}, 1 \mathrm{H}), 4.11(\mathrm{~s}, 1 \mathrm{H}), 4.08-3.89(\mathrm{~m}$, $5 \mathrm{H}), 3.89-3.75(\mathrm{~m}, 3 \mathrm{H}), 3.71(\mathrm{~d}, \mathrm{~J}=9.5 \mathrm{~Hz}, 1 \mathrm{H}), 3.62(\mathrm{t}, \mathrm{J}=9.7$ $\mathrm{Hz}, 1 \mathrm{H}), 3.50$ (dd, J=19.7, $12.6 \mathrm{~Hz}, 1 \mathrm{H}) .{ }^{13} \mathrm{C}$ NMR $(100 \mathrm{MHz}$, $\left.\mathrm{D}_{2} \mathrm{O}\right): \delta=102.6,102.5,96.2,96.0,79.2,78.9,78.8,77.2,75.2,74.1$, 73.3, 73.0, 72.9, 72.6, 71.3, 69.1, 63.4, 62.9. ESI-MS: $m / z 386.6$ [M+HCOO] $]^{-}$. HRMS: calcd for $\mathrm{C}_{12} \mathrm{H}_{21} \mathrm{O}_{11} 341.1084$, found: 341.1076. $[\mathrm{a}]_{\mathrm{D}}{ }^{21}=-2.8,\left(\mathrm{c} 0.4, \mathrm{H}_{2} \mathrm{O}\right)$.

Benzyl 2,3-di-O-benzyl-4,6-O-benzylidene- $\beta$-D-mannopyranosyl$(1 \rightarrow 4)-2,3-d i-O-b e n z y l-6-O-a c e t y l-\beta-D-m a n n o p y r a n o s y l-(1 \rightarrow 4)-2,3-$ di-O-benzyl-6-O-acetyl- $\beta$-D-mannopyranoside (7)

Sulfoxide 1 was coupled with compound 5 using the standard sulfoxide $\beta$-mannosylation protocol to afford the title compound in $65 \%$ yield as a colorless oil. ${ }^{1} \mathrm{H}$ NMR $(400 \mathrm{MHz}$, $\left.\mathrm{CDCl}_{3}\right) \delta$ 7.62-6.96 (m, 40H), $5.51(\mathrm{~s}, 1 \mathrm{H}), 4.99-4.90(\mathrm{~m}, 2 \mathrm{H})$, 4.88-4.68 (m, 7H), 4.65-4.48 (m, 7H), $4.44(\mathrm{~s}, 1 \mathrm{H}), 4.33(\mathrm{~m}, 2 \mathrm{H})$, 4.14-3.92 (m, 6H), 3.91-3.82 (m, 3H), $3.61(\mathrm{t}, \mathrm{J}=10.2 \mathrm{~Hz}, 1 \mathrm{H})$, $3.56-3.43(\mathrm{~m}, 4 \mathrm{H}), 3.26(\mathrm{~m}, 1 \mathrm{H}), 3.07(\mathrm{td}, \mathrm{J}=9.7,4.9 \mathrm{~Hz}, 1 \mathrm{H})$, $2.08(\mathrm{~s}, 3 \mathrm{H}), 1.90(\mathrm{~s}, 3 \mathrm{H}) .{ }^{13} \mathrm{C} \mathrm{NMR}\left(100 \mathrm{MHz}, \mathrm{CDCl}_{3}\right): \delta=170.8$, 170.6, 138.7, 138.6, 138.5, 139.4, 139.3, 137.5, 137.1, 128.8, 128.4-127.1, 126.1, 101.9, 101.3, 100.8, 99.87, 79.8, 79.6, 78.50, 78.2, 77.2, 75.7, 75.4, 75.2, 74.3, 74.0, 73.9, 73.3, 73.1, 72.5, 72.0, 71.4, 70.7, 68.4, 67.4, 63.4, 63.1, 20.9, 20.7. ESI-MS: $m / z$ 1329.7 $[\mathrm{M}+\mathrm{Na}]^{+}$. HRMS: calcd for $\mathrm{C}_{78} \mathrm{H}_{82} \mathrm{O}_{18} \mathrm{Na} 1329.5399$, found: 1329.5417. $[\mathrm{\alpha}]_{\mathrm{D}}{ }^{21}=-66.3,\left(\mathrm{c} 1, \mathrm{CHCl}_{3}\right)$.

Benzyl 2,3-di-O-benzyl-6-O-acetyl- $\beta$-D-mannopyranosyl-(1 $\rightarrow 4)$ 2,3-di-O-benzyl-6-O-acetyl- $\beta$-D-mannopyranosyl-(1 $\rightarrow 4)-2,3-d i-0$ benzyl-6-0-acetyl- $\beta$-D-mannopyranoside (8)

The method used to prepare 3 was employed for the synthesis of the title compound (colorless oil, $81 \%$ yield) using compound 7 as the starting material. ${ }^{1} \mathrm{H}$ NMR $\left(400 \mathrm{MHz}, \mathrm{CDCl}_{3}\right)$

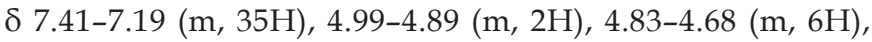
$4.64-4.50(\mathrm{~m}, 6 \mathrm{H}), 4.48(\mathrm{~s}, 1 \mathrm{H}), 4.46-4.28(\mathrm{~m}, 4 \mathrm{H}), 4.21-4.10(\mathrm{~m}$, $4 \mathrm{H}), 4.08-3.96(\mathrm{~m}, 2 \mathrm{H}), 3.90(\mathrm{~m}, 1 \mathrm{H}), 3.87-3.79(\mathrm{~m}, 3 \mathrm{H}), 3.53$ $(\mathrm{m}, 3 \mathrm{H}), 3.35(\mathrm{dd}, \mathrm{J}=8.1,4.2 \mathrm{~Hz}, 1 \mathrm{H}), 3.21(\mathrm{dd}, \mathrm{J}=9.4,2.6 \mathrm{~Hz}$, 1H), 3.16-3.08 (m, 1H), 2.55 (s, 1H), 2.07 (s, 3H), $1.91(\mathrm{~s}, 3 \mathrm{H})$, $1.90(\mathrm{~s}, 3 \mathrm{H}) .{ }^{13} \mathrm{C}$ NMR $\left(100 \mathrm{MHz}, \mathrm{CDCl}_{3}\right): \delta=171.2,170.7,170.6$, 138.6, 138.5, 138.4, 138.3, 137.5, 137.0, 128.5-127.1, 101.3, 100.7, 99.8, 81.3, 79.5, 79.4, 77.2, 75.4, 75.2, 75.0, 74.4, 74.2, 74.1, 74.0, 73.9, 73.8, 73.3, 73.1, 71.7, 71.4, 71.3, 70.6, 66.2, 63.5, 63.4, 63.2, 20.9, 20.7, 20.6. ESI-MS: $m / z 1283.6[\mathrm{M}+\mathrm{Na}]^{+}$. HRMS: calcd for $\mathrm{C}_{73} \mathrm{H}_{80} \mathrm{O}_{19} \mathrm{Na} 1283.5192$, found: 1283.5153 . [ $\left.\mathrm{a}\right]_{\mathrm{D}}{ }^{21}=-79.5$, (c 1 , $\mathrm{CHCl}_{3}$ ).

\section{$\beta-(1,4)-D$-Mannotriose $(9)^{[22]}$}

The method used to prepare 6 was employed for the synthesis of the title compound (white amorphous solid, $63 \%$ yield) using compound 8 as the starting material. ${ }^{1} \mathrm{H}$ NMR (400 $\left.\mathrm{MHz}, \mathrm{D}_{2} \mathrm{O}\right) \delta 5.21(\mathrm{~s}, 0.53 \mathrm{H}), 4.93(\mathrm{~s}, 0.47 \mathrm{H}), 4.79(\mathrm{~s}, 1 \mathrm{H}), 4.76$ $(\mathrm{d}, \mathrm{J}=6.1 \mathrm{~Hz}, 1 \mathrm{H}), 4.16(\mathrm{~s}, 1 \mathrm{H}), 4.09(\mathrm{~d}, \mathrm{~J}=2.5 \mathrm{~Hz}, 1 \mathrm{H}), 4.05-3.87$ $(\mathrm{m}, 6 \mathrm{H}), 3.85(\mathrm{~d}, \mathrm{~J}=6.1 \mathrm{~Hz}, 2 \mathrm{H}), 3.82-3.74(\mathrm{~m}, 3 \mathrm{H}), 3.71(\mathrm{dd}$, $\mathrm{J}=16.6,6.0 \mathrm{~Hz}, 2 \mathrm{H}), 3.59(\mathrm{t}, \mathrm{J}=9.4 \mathrm{~Hz}, 2 \mathrm{H}), 3.49$ (dd, J=20.6, 13.3 $\mathrm{Hz}, 1 \mathrm{H}) .{ }^{13} \mathrm{C}$ NMR $\left(100 \mathrm{MHz}, \mathrm{D}_{2} \mathrm{O}\right): \delta=94.8,94.7,94.6,88.5$, 88.3, 71.8-71.1, 69.7, 69.4, 67.4, 66.3, 66.2, 66.1, 65.5, 65.2, 64.8, 64.6, 64.1, 63.6, 61.3, 57.7, 57.0, 55.7, 55.4, 55.2, 55.1. ESI-MS: $\mathrm{m} / \mathrm{z} 549.0$ [M+HCOO] $]^{-}$. HRMS: calcd for $\mathrm{C}_{18} \mathrm{H}_{31} \mathrm{O}_{16} 503.1612$, found: 503.1605. $[\mathrm{a}]_{\mathrm{D}}{ }^{21}=-22.5,\left(\mathrm{c} 0.4, \mathrm{H}_{2} \mathrm{O}\right)$.

Benzyl 2,3-di-O-benzyl-4,6-O-benzylidene- $\beta$-D-mannopyranosyl$(1 \rightarrow 4)-2,3-d i-O-b e n z y l-6-0$-acetyl- $\beta$ - $D$-mannopyranosyl-(1 $\rightarrow 4)$ 2,3-di-O-benzyl-6-O-acetyl- $\beta-D$-mannopyranosyl-(1 $\rightarrow 4)-2,3-d i-0$ benzyl-6-O-acetyl- $\beta$ - $D$-mannopyranoside (10)

Sulfoxide 1 was coupled with compound 8 using the standard sulfoxide $\beta$-mannosylation protocol to afford the title compound in $68 \%$ yield as a colorless oil. ${ }^{1} \mathrm{H}$ NMR $(400 \mathrm{MHz}$, $\left.\mathrm{CDCl}_{3}\right) \delta 7.32(\mathrm{mz}, 50 \mathrm{H}), 5.50(\mathrm{~s}, 1 \mathrm{H}), 4.94(\mathrm{dd}, \mathrm{J}=16.4,12.2 \mathrm{~Hz}$, $2 \mathrm{H}), 4.87-4.66(\mathrm{~m}, 10 \mathrm{H}), 4.66-4.51(\mathrm{~m}, 8 \mathrm{H}), 4.43(\mathrm{~m}, 2 \mathrm{H}), 4.36-$ $4.25(\mathrm{~m}, 2 \mathrm{H}), 4.10(\mathrm{~m}, 6 \mathrm{H}), 4.03-3.91(\mathrm{~m}, 4 \mathrm{H}), 3.88(\mathrm{dd}, \mathrm{J}=9.1$, $2.8 \mathrm{~Hz}, 2 \mathrm{H}), 3.82(\mathrm{dd}, \mathrm{J}=7.1,2.4 \mathrm{~Hz}, 2 \mathrm{H}), 3.60(\mathrm{t}, \mathrm{J}=10.3 \mathrm{~Hz}, 1 \mathrm{H})$, 3.56-3.48 (m, 4H), $3.26(\mathrm{~m}, 2 \mathrm{H}), 3.13-2.98(\mathrm{td}, \mathrm{J}=9.9,4.9 \mathrm{~Hz}$, $1 \mathrm{H}), 2.06(\mathrm{~s}, 3 \mathrm{H}), 1.89(2 \mathrm{~s}, 6 \mathrm{H}) .{ }^{13} \mathrm{C} \mathrm{NMR}\left(100 \mathrm{MHz}, \mathrm{CDCl}_{3}\right)$ : $\delta=171.0,170.8,170.7,138.8,138.7,138.6,138.5,138.4,137.6$, 137.2, 128.9, 128.5-127.1, 126.2, 102.0, 101.4, 101.3, 100.8, 99.9, $80.1,79.9,79.5,78.6,78.3,75.7,75.6,75.5,75.3,75.2,74.4,74.3$, 74.1, 74.0, 73.4, 73.3, 73.2, 72.6, 72.1, 72.0, 71.5, 70.8, 68.5, 67.5, 63.7, 63.2, 50.9, 21.0, 20.9, 20.8. ESI-MS: $m / z 1713.3[\mathrm{M}+\mathrm{Na}]^{+}$. $[\mathrm{a}]_{\mathrm{D}}{ }^{21}=-72.5,\left(\mathrm{c} 1, \mathrm{CHCl}_{3}\right)$.

Benzyl 2,3-di-O-benzyl-6-0-acetyl- $\beta$-D-mannopyranosyl-(1 $\rightarrow 4)$ 2,3-di-0-benzyl-6-O-acetyl- $\beta$-D-mannopyranosyl-(1 $\rightarrow 4$ )-2,3-di-0benzyl-6-O-acetyl- $\beta$-D-mannopyranosyl-(1 $\rightarrow 4)$-2,3-di-0-benzyl-60 -acetyl- $\beta$-D-mannopyranoside (11)

The method used to prepare 3 was employed for the synthesis of the title compound (colorless oil, $80 \%$ yield) using compound 10 as the starting material. ${ }^{1} \mathrm{H}$ NMR $\left(400 \mathrm{MHz}, \mathrm{CDCl}_{3}\right)$

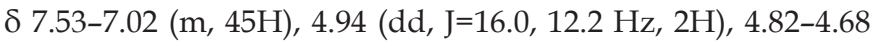
$(\mathrm{m}, 9 \mathrm{H}), 4.55(\mathrm{~m}, 9 \mathrm{H}), 4.45-4.27(\mathrm{~m}, 4 \mathrm{H}), 4.20-4.12(\mathrm{~m}, 4 \mathrm{H})$, 4.11-3.91 (m, 5H), $3.87(\mathrm{dd}, \mathrm{J}=9.4,2.8 \mathrm{~Hz}, 2 \mathrm{H}), 3.85-3.78(\mathrm{~m}$, $3 \mathrm{H}), 3.50(\mathrm{~m}, 4 \mathrm{H}), 3.35(\mathrm{~m}, 1 \mathrm{H}), 3.28(\mathrm{~m}, 1 \mathrm{H}), 3.21(\mathrm{dd}, \mathrm{J}=9.4$, $2.7 \mathrm{~Hz}, 1 \mathrm{H}), 3.14-3.08(\mathrm{~m}, 1 \mathrm{H}), 2.49(\mathrm{~s}, 1 \mathrm{H}), 2.06(\mathrm{~s}, 3 \mathrm{H}), 1.90$ $(\mathrm{s}, 3 \mathrm{H}), 1.89(\mathrm{~s}, 3 \mathrm{H}), 1.88(\mathrm{~s}, 3 \mathrm{H}) .{ }^{13} \mathrm{C}$ NMR $\left(100 \mathrm{MHz}, \mathrm{CDCl}_{3}\right)$ : $\delta=171.3,170.7,170.6,170.5,138.7-138.3,137.6,137.1,128.5-$ 127.2, 101.4, 101.2, 100.7, 99.9, 81.3, 79.9, 79.5, 79.4, 77.2, 75.5, 75.3, 75.2, 75.0, 74.4, 74.3, 74.2, 74.1, 74.0, 73.9, 73.8, 73.3, 73.1, 71.9, 71.7, 71.4, 71.3, 70.7, 66.2, 63.5, 63.4, 63.1, 29.6, 20.9, 20.7, 20.6. ESI-MS: $m / z 1667.8[\mathrm{M}+\mathrm{Na}]^{+} .[\mathrm{a}]_{\mathrm{D}}{ }^{21}=-79.2,\left(\mathrm{c} 1, \mathrm{CHCl}_{3}\right)$.

\section{$\beta-(1,4)-D-M a n n o t e t r a o s e(12)^{[23]}$}

The method used to prepare 9 was employed for the synthesis of the title compound (white amorphous solid, 50\% yield) using compound $\mathbf{1 1}$ as the starting material. ${ }^{1} \mathrm{H}$ NMR (400 $\left.\mathrm{MHz}, \mathrm{D}_{2} \mathrm{O}\right) \delta 5.05(\mathrm{~s}, 0.57 \mathrm{H}), 4.79(\mathrm{~s}, 0.43 \mathrm{H}), 4.62(\mathrm{~d}, \mathrm{~J}=13.3 \mathrm{~Hz}$, $3 \mathrm{H}), 4.00(\mathrm{~s}, 2 \mathrm{H}), 3.94(\mathrm{~d}, \mathrm{~J}=2.1 \mathrm{~Hz}, 1 \mathrm{H}), 3.89-3.83(\mathrm{~m}, 2 \mathrm{H}), 3.80$ 
(d, J=13.1 Hz, 4H), 3.71 (d, J=20.3 Hz, 5H), 3.67-3.57 (m, 4H), 3.57-3.51 (m, 1H), $3.44(\mathrm{t}, \mathrm{J}=9.1 \mathrm{~Hz}, 3 \mathrm{H}), 3.40-3.26(\mathrm{~m}, 2 \mathrm{H}) .{ }^{13} \mathrm{C}$ NMR (100 MHz, D 2 ): $\delta=100.1-100.0,93.8,93.6,76.9-76.3$, 74.9, 74.7, 72.7, 71.6, 71.4, 70.8, 70.6, 70.5, 70.4, 70.1, 70.0, 69.8, 68.8, 66.6, 60.9, 60.5. ESI-MS: $m / z 710.3$ [M+HCOO] ${ }^{-}$. HRMS: calcd for $\mathrm{C}_{24} \mathrm{H}_{42} \mathrm{O}_{21} \mathrm{Na}$ 689.2116, found: 689.2144. [a $]_{\mathrm{D}}{ }^{21}=-18.7$, (c $0.3, \mathrm{H}_{2} \mathrm{O}$ ).

Benzyl 2,3-di-O-benzyl-4,6-0-benzylidene- $\beta$ - $D$-mannopyranosyl$(1 \rightarrow 4)-2,3-d i-0-b e n z y l-6-0$-acetyl- $\beta$ - $D$-mannopyranosyl-( $1 \rightarrow 4)$ 2,3-di-0-benzyl-6-0-acetyl- $\beta$-D-mannopyranosyl-(1 $\rightarrow 4)-2,3-d i-0$ benzyl-6-0-acetyl- $\beta$-D-mannopyranosyl-(1 $\rightarrow$ 4)-2,3-di-0-benzyl-6-0acetyl- $\beta$-D-mannopyranoside (13)

Sulfoxide 1 was coupled with compound 11 using the standard sulfoxide $\beta$-mannosylation protocol to afford the title compound in $75 \%$ yield as a colorless oil. ${ }^{1} \mathrm{H}$ NMR $(400 \mathrm{MHz}$, $\left.\mathrm{CDCl}_{3}\right) \delta$ 7.51-7.14 (m, 60H), 5.51 (s, 1H), 4.95 (dd, J=16.1, 12.2 $\mathrm{Hz}, 2 \mathrm{H}), 4.89-4.66(\mathrm{~m}, 12 \mathrm{H}), 4.65-4.48(\mathrm{~m}, 11 \mathrm{H}), 4.44(\mathrm{~d}, \mathrm{~J}=7.2$ $\mathrm{Hz}, 1 \mathrm{H}), 4.41-4.23(\mathrm{~m}, 3 \mathrm{H}), 4.21-4.00(\mathrm{~m}, 8 \mathrm{H}), 3.96(\mathrm{~m}, 4 \mathrm{H}), 3.88$ $(\mathrm{m}, 2 \mathrm{H}), 3.85-3.78(\mathrm{~m}, 3 \mathrm{H}), 3.61(\mathrm{t}, \mathrm{J}=10.3 \mathrm{~Hz}, 1 \mathrm{H}), 3.57-3.40$ $(\mathrm{m}, 6 \mathrm{H}), 3.34-3.21(\mathrm{~m}, 3 \mathrm{H}), 3.07(\mathrm{td}, \mathrm{J}=9.7,4.9 \mathrm{~Hz}, 1 \mathrm{H}), 2.06(\mathrm{~s}$, $3 \mathrm{H}), 1.95-1.82(3 \mathrm{~s}, 9 \mathrm{H}) .{ }^{13} \mathrm{C} \mathrm{NMR}\left(100 \mathrm{MHz}, \mathrm{CDCl}_{3}\right): \delta=170.7$, $170.6-170.5,138.7,138.5,138.4,138.3,137.5,137.1,128.8,128.4-$ 127.0, 126.0, 101.8, 101.3, 101.1, 100.6, 99.8, 80.0, 79.9, 79.8, 79.4, $78.5,78.2,77.2,75.6,75.5,75.4,75.2,75.1,75.0,74.3,74.2,74.0$, 73.8, 73.3, 73.1, 72.4, 72.0, 71.9, 71.8, 71.4, 70.7, 68.4, 67.3, 63.6, 63.1, 29.7, 20.9, 20.7. ESI-MS: $m / z 2099.6[\mathrm{M}+\mathrm{Na}]^{+} .[a]_{\mathrm{D}}{ }^{21}=-64.7$, (c $1, \mathrm{CHCl}_{3}$ ).

Benzyl 2,3-di-O-benzyl-6-0-acetyl- $\beta-D-m a n n o p y r a n o s y l-(1 \rightarrow 4)-$ 2,3-di-O-benzyl-6-O-acetyl- $\beta$-D-mannopyranosyl-(1 $\rightarrow$ 4)-2,3-di-Obenzyl-6-0-acetyl- $\beta$-D-mannopyranosyl-(1 $\rightarrow 4)-2,3-d i-0-$ benzyl-6-0acetyl- $\beta$-D-mannopyranosyl-(1 $\rightarrow 4)-2,3-d i-0-$ benzyl-6-O-acetyl- $\beta-D$ mannopyranoside (14)

The method used to prepare 3 was employed for the synthesis of the title compound (colorless oil, 65\% yield) using compound 13 as the starting material. ${ }^{1} \mathrm{H} \mathrm{NMR}\left(400 \mathrm{MHz}, \mathrm{CDCl}_{3}\right)$

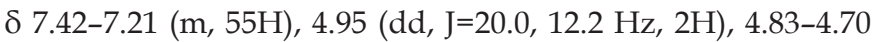
$(\mathrm{m}, 12 \mathrm{H}), 4.56(\mathrm{ddd}, \mathrm{J}=31.6,16.6,10.2 \mathrm{~Hz}, 11 \mathrm{H}), 4.47-4.28(\mathrm{~m}$, $5 \mathrm{H}), 4.21-4.10(\mathrm{~m}, 7 \mathrm{H}), 4.08-4.04(\mathrm{~m}, 1 \mathrm{H}), 4.01$ (d, J=9.1 Hz, $1 \mathrm{H}), 3.99-3.93$ (m, 2H), 3.89 (dd, J=13.4, $2.4 \mathrm{~Hz}, 2 \mathrm{H}), 3.86-3.78$ $(\mathrm{m}, 4 \mathrm{H}), 3.57-3.46(\mathrm{~m}, 5 \mathrm{H}), 3.39-3.33(\mathrm{~m}, 1 \mathrm{H}), 3.33-3.26(\mathrm{~m}$, 2H), 3.22 (dd, J=9.4, $2.4 \mathrm{~Hz}, 1 \mathrm{H}), 3.15-3.09$ (m, 1H), 2.50 (s, 1H), 2.07 (s, 3H), 1.96-1.85 (4s, 12H). $\left.{ }^{13} \mathrm{C} \mathrm{NMR} \mathrm{(100} \mathrm{MHz,} \mathrm{CDCl}_{3}\right)$ : $\delta=171.3,170.7-170.5,138.7-138.3,137.6,137.1,128.5-127.1$, 101.3, 101.2, 101.1, 100.5, 99.8, 81.3, 79.9, 79.8, 79.5, 79.3, 77.2, $75.4,75.3,75.1,75.0,74.4,74.3,74.1,74.0,73.9,73.8,73.3,73.1$, 71.8, 71.7, 71.4, 71.3, 70.7, 66.2, 63.5, 63.4, 63.1, 29.6, 20.9, 20.7, 20.6. ESI-MS: $m / z 2054.8[\mathrm{M}+\mathrm{Na}]^{+} .[a]_{\mathrm{D}}{ }^{21}=-67.0$, (c $\left.1, \mathrm{CHCl}_{3}\right)$.

\section{$\beta-(1,4)-D-M a n n o p e n t o s e(15)^{[24]}$}

The method used to prepare 9 was employed for the synthesis of the title compound (white amorphous solid, 33\% yield) using compound $\mathbf{1 4}$ as the starting material. ${ }^{1} \mathrm{H}$ NMR (400 $\left.\mathrm{MHz}, \mathrm{D}_{2} \mathrm{O}\right) \delta 5.08(\mathrm{~s}, 0.60 \mathrm{H}), 4.81(\mathrm{~s}, 0.40 \mathrm{H}), 4.71(\mathrm{~d}, \mathrm{~J}=6.4 \mathrm{~Hz}$, $2 \mathrm{H}), 4.57$ (d, J=6.4 Hz, 2H), 4.02 (s, 3H), $3.96(\mathrm{~d}, \mathrm{~J}=3.1 \mathrm{~Hz}, 1 \mathrm{H})$, $3.85(\mathrm{dd}, \mathrm{J}=18.0,8.6 \mathrm{~Hz}, 7 \mathrm{H}), 3.68(\mathrm{dt}, \mathrm{J}=12.3,10.6 \mathrm{~Hz}, 12 \mathrm{H})$, $3.56(\mathrm{dd}, \mathrm{J}=14.3,4.9 \mathrm{~Hz}, 2 \mathrm{H}), 3.45$ (d, J=9.1 Hz, 4H), 3.35 (d, $\mathrm{J}=7.2 \mathrm{~Hz}, 1 \mathrm{H}) .{ }^{13} \mathrm{C}$ NMR $\left(100 \mathrm{MHz}, \mathrm{D}_{2} \mathrm{O}\right): \delta=100.1-100.0,93.7$, 93.6, 76.7, 76.6-76.3, 74.9, 72.7, 71.6, 71.4, 71.3, 70.8, 70.6, 70.4, 70.1, 69.9, 69.8, 69.7, 68.8, 66.6, 60.9, 60.5. ESI-MS: $m / z 872.5$ $\left[\mathrm{M}+\mathrm{HCOO}^{-}\right.$. HRMS: calcd for $\mathrm{C}_{30} \mathrm{H}_{51} \mathrm{O}_{26} 827.2669$, found: 827.2680. $[\mathrm{a}]_{\mathrm{D}}{ }^{21}=-27.0$, (c $\left.0.2, \mathrm{H}_{2} \mathrm{O}\right)$.

Benzyl 2,3-di-O-benzyl-4,6-0-benzylidene- $\beta$-D-mannopyranosyl$(1 \rightarrow 4)-2,3-d i-0$-benzyl-6-0-acetyl- $\beta$ - $D$-mannopyranosyl-(1 $\rightarrow 4)$ 2,3-di-0-benzyl-6-0-acetyl- $\beta$ - $D$-mannopyranosyl-(1 $\rightarrow 4)-2,3-d i-0$ benzyl-6-0-acetyl- $\beta$-D-mannopyranosyl-(1 $\rightarrow 4)-2,3-d i-0-b e n z y l-6-0$ acetyl- $\beta$-D-mannopyranosyl-(1 $\rightarrow 4)-2,3-$ di-O-benzyl-6-0-acetyl- $\beta-D$ mannopyranoside (16)

Sulfoxide 1 was coupled with compound 14 using the standard sulfoxide $\beta$-mannosylation protocol to afford the title compound in $60 \%$ yield as a colorless oil. ${ }^{1} \mathrm{H}$ NMR $(400 \mathrm{MHz}$, $\left.\mathrm{CDCl}_{3}\right) \delta 7.47-7.17(\mathrm{~m}, 70 \mathrm{H}), 5.50(\mathrm{~s}, 1 \mathrm{H}), 4.94(\mathrm{dd}, \mathrm{J}=16.4$, $12.2 \mathrm{~Hz}, 2 \mathrm{H}), 4.87-4.76$ (m, 2H), 4.76-4.67 (m, 13H), 4.64-4.47 $(\mathrm{m}, 14 \mathrm{H}), 4.43(\mathrm{~s}, 1 \mathrm{H}), 4.39-4.26(\mathrm{~m}, 2 \mathrm{H}), 4.17-4.03(\mathrm{~m}, 9 \mathrm{H})$, 4.02-3.90 (m, 6H), $3.87(\mathrm{dd}, \mathrm{J}=8.3,3.1 \mathrm{~Hz}, 2 \mathrm{H}), 3.83-3.76(\mathrm{~m}$, $4 \mathrm{H}), 3.60(\mathrm{t}, \mathrm{J}=10.2 \mathrm{~Hz}, 1 \mathrm{H}), 3.55-3.43(\mathrm{~m}, 7 \mathrm{H}), 3.33-3.21(\mathrm{~m}$, $4 \mathrm{H}), 3.06(\mathrm{td}, \mathrm{J}=9.7,4.8 \mathrm{~Hz}, 1 \mathrm{H}), 2.05(\mathrm{~s}, 3 \mathrm{H}), 1.88(4 \mathrm{~s}, 12 \mathrm{H}) .{ }^{13} \mathrm{C}$ NMR $\left(100 \mathrm{MHz}, \mathrm{CDCl}_{3}\right): \delta=170.8,170.7-170.6,138.7,138.5$, 138.4, 138.3, 137.5, 137.1, 128.8, 128.4-127.0, 126.0, 101.8, 101.3, 101.1, 100.7, 100.5, 99.8, 80.0, 79.9, 79.8, 79.3, 78.4, 78.2, 77.2, 75.6, 75.5-75.1, 74.3, 74.2, 74.0, 73.9, 73.3, 73.1, 72.4, 72.0, 71.9, 71.8, 71.4, 70.7, 68.4, 67.3, 63.5, 63.1, 29.6, 20.9, 20.7. ESI-MS: $\mathrm{m} / \mathrm{z} 2482.9[\mathrm{M}+\mathrm{Na}]^{+} .[\mathrm{a}]_{\mathrm{D}}{ }^{21}=-68.8,\left(\mathrm{c} 0.4, \mathrm{CHCl}_{3}\right)$.

\section{$\beta-(1,4)-D-M a n n o h e p t a o s e(17)$}

The method used to prepare 9 was employed for the synthesis of the title compound (white amorphous solid, 35\% yield) using compound $\mathbf{1 6}$ as the starting material. ${ }^{1} \mathrm{H}$ NMR (400 $\left.\mathrm{MHz}, \mathrm{D}_{2} \mathrm{O}\right) \delta 5.06(\mathrm{~s}, 0.60 \mathrm{H}), 4.79(\mathrm{~s}, 0.40 \mathrm{H}), 4.62(\mathrm{~d}, \mathrm{~J}=11.9$ $\mathrm{Hz}, 5 \mathrm{H}), 4.01(\mathrm{~s}, 3 \mathrm{H}), 3.93(\mathrm{t}, \mathrm{J}=10.6 \mathrm{~Hz}, 2 \mathrm{H}), 3.82(\mathrm{dt}, \mathrm{J}=29.0$, $12.0 \mathrm{~Hz}, 8 \mathrm{H}), 3.75-3.57(\mathrm{~m}, 14 \mathrm{H}), 3.54(\mathrm{dd}, \mathrm{J}=16.2,6.8 \mathrm{~Hz}, 2 \mathrm{H})$, $3.43(\mathrm{~d}, \mathrm{~J}=9.0 \mathrm{~Hz}, 4 \mathrm{H}), 3.41-3.27(\mathrm{~m}, 3 \mathrm{H}) .{ }^{13} \mathrm{C} \mathrm{NMR}(100 \mathrm{MHz}$, $\left.\mathrm{D}_{2} \mathrm{O}\right): \delta=100.1-100.0,93.8,93.6,76.7,76.6-76.3,75.0,72.7,71.6$, 71.5-71.3, 70.8, 70.6, 70.4, 70.2, 69.9-69.8, 68.9, 66.6, 60.9, 60.4. ESI-MS: $m / z$ 1034.7 [M+HCOO] ${ }^{-}$. HRMS: calcd for $\mathrm{C}_{36} \mathrm{H}_{61} \mathrm{O}_{31}$ 989.3197, found: 989.3218. [a $]_{\mathrm{D}}{ }^{21}=-15.5$, (c 0.4, $\left.\mathrm{H}_{2} \mathrm{O}\right)$.

\section{Acknowledgements}

This work was supported by the National Science \& Technology Major Project "Key New Drug Creation and Manufacturing Program," China (2012ZX09301001).

\section{Author contribution}

Jing-kang SHEN and Mei-yu GENG designed research; Ruwei JIANG, Xiao-guang DU, Xuan ZHANG, Ding-yu HU, Tao MENG, and Yue-lei CHEN performed research; Ru-wei JIANG, Yue-lei CHEN, Mei-yu GENG, and Jing-kang SHEN analyzed data; Ru-wei JIANG, Xiao-guang DU, Yue-lei CHEN, Mei-yu GENG, and Jing-kang SHEN wrote the paper. 


\section{References}

1 Wimo A, Winblad B, Jonsson L. The worldwide societal costs of dementia: Estimates for 2009. Alzheimers Dement 2010; 6: 98-103.

2 Sloane PD, Zimmerman S, Suchindran C, Reed P, Wang L, Boustani M, et al. The public health impact of Alzheimer's disease, 2000-2050: potential implication of treatment advances. Annu Rev Public Health 2002; 23: 213-31.

3 Cras P, Kawai M, Lowery D, Gonzalez DP, Greenberg B, Perry G. Senile plaque neurites in Alzheimer disease accumulate amyloid precursor peptide. Proc Natl Acad Sci U S A 1991; 88: 7552-6.

4 Blanquet V, Goldgaber D, Turleau C, Creau GN, Delabar J, Sinet PM, et al. The beta amyloid peptide (AD-AP) cDNA hybridizes in normal and Alzheimer individuals near the interface of 21q21 and q22.1. Ann Genet 1987; 30: 68-9.

5 Ove A. The bittersweet promise of glycobiology. Nat Biotechnol 2001; 19: $913-7$.

6 Sasaki T, Takasuka N. Further study of the structure of lentinan, an anti-tumor polysaccharide from Lentinus edodes. Carbohydr Res 1976; 47: 99-104.

7 Schaefer DJ, Krylov VS. Anti-HIV activity of extracts and compounds from algae and cyanobacteria. Ecotoxicol Environ Saf 2000; 45: 208-27.

8 Kim HM, Kang JS, Kim JY. Evaluation of antidiabetic activity of polysaccharide isolated from Phellinus linteus in non-obese diabetic mouse. Int Immunopharmacol 2010; 10: 72-8.

9 Ho YS, Yu MS, Yang XF, So KF, Yuen WH, Chang RC. Neuroprotective effects of polysaccharides from wolfberry, the fruits of Lycium barbarum, against homocysteine-induced toxicity in rat cortical neurons. J Alzhiermers Dis 2010; 19: 813-27.

10 Townsend M, Cleary JP, Mehta T, Hofmeister J, Lesne S, Selkoe DJ, et al. Orally available compound prevents deficits in memory caused by the Alzheimer amyloid- $\beta$ oligomers. Ann Neurol 2006; 60: 668-76.

11 Geng MY. Protective effect of marine-derived oligosaccharide as a candidate anti-AD drug and its related mechanisms. Chin Pharmacologist 2007; 24: 7.

12 Geng MY, Xin XL, Sun GQ. Preparation of brown algae oligosaccharide and its derivatives and their uses. CN 200580009396.5.
13 Crich D, Sun SX. Formation of beta-mannopyranosides of primary alcohols using the sulfoxide method. J Org Chem 1996; 61: 4506-7.

14 Crich D, Sun SX. Direct synthesis of beta-mannopyranosides by the sulfoxide method. J Org Chem 1997; 62: 1198-9.

15 Crich D, Mataka J, Sun SX, Lam KC, Rheingold AL, Wink DJ. Stereoselective sulfoxidation of alpha-mannopyranosyl thioglycosides: The exo-anomeric effect in action. Chem Commun 1998; 24: 2763-4.

16 Crich D, Mark S, Yao QJ, John P. 2,4,6-Tri-tert-butylpyrimidine (TTBP): A cost effective, readily available alternative to the hindered base 2,6-ditert-butylpyridine and its 4-substituted derivatives in glycosylation and other reactions. Synth Stuttgart 2000; 2: 323-6.

17 Crich D, Li WJ, Li HM. Direct chemical synthesis of the beta-mannans: Linear and block syntheses of the alternating beta- $(l \rightarrow 3)$-beta- $(l \rightarrow 4)$ mannan common to Rhodotorula glutinis, Rhodotorula mucilaginosa, and Leptospira biflexa. J Am Chem Soc 2004; 126: 15081-6.

18 Crich D, Sun SX. Direct chemical synthesis of beta-mannopyranosides and other glycosides via glycosyl triflates. Tetrahedron 1998; 54: 8321-48.

19 Jalsa NK, Singh G. A unique approach to the synthesis of a dengue vaccine and the novel tetrasaccharide that results. TetrahedronAsymmetry 2009; 20: 867-74.

20 Bock K, Pedersen C. A study of ${ }^{13} \mathrm{CH}$ coupling constants in hexopyranoses. J Chem Soc Perkin Trans 2 1974; 3: 293-7.

21 Ikeda R, Maeda T. Structural studies of the capsular polysaccharide of a non-neoformans Cryptococcus species identified as C-laurentii, which was reclassified as Cryptococcus flavescens, from a patient with AIDS. Carbohydr Res 2004; 339: 503-9.

22 Chow JT, Williamson DA, Yates KM, Goux WJ. Chemical characterization of the immunomodulating polysaccharide of Aloe vera $L$. Carbohydr Res 2005; 340: 1131-42.

23 Issa MA. Structural investigation of water-hyacinth (EichhorniaCrassipes) polysaccharides .1. Water-Soluble Polysaccharides. Can J Chem 1988; 66: 2777-81.

24 Fujimoto $\mathrm{H}$, Isomura M, Ajisaka K. Syntheses of alkyl beta- $D$ mannopyranosides and beta-1,4-linked oligosaccharides using betamannosidase from Rhizopus niveus. Biosci Biotech Bioch 1997; 61: 164-5. 